\title{
HAPIscreen, a method for high-throughput aptamer identification
}

\author{
Eric Dausse ${ }^{1,3+}$, Saïd Taouji ${ }^{2,3 \dagger}$, Laetitia Evadé ${ }^{1,3}$, Carmelo Di Primo ${ }^{1,3}$, Eric Chevet ${ }^{2,3^{*}}$ and Jean-Jacques Toulmé ${ }^{1,3^{*}}$
}

\begin{abstract}
Background: Aptamers are oligonucleotides displaying specific binding properties for a predetermined target. They are selected from libraries of randomly synthesized candidates through an in vitro selection process termed SELEX (Systematic Evolution of Ligands by EXponential enrichment) alternating selection and amplification steps. SELEX is followed by cloning and sequencing of the enriched pool of oligonucleotides to enable comparison of the selected sequences. The most represented candidates are then synthesized and their binding properties are individually evaluated thus leading to the identification of aptamers. These post-selection steps are time consuming and introduce a bias to the expense of poorly amplified binders that might be of high affinity and are consequently underrepresented. A method that would circumvent these limitations would be highly valuable.
\end{abstract}

Results: We describe a novel homogeneous solution-based method for screening large populations of oligonucleotide candidates generated from SELEX. This approach, based on the AlphaScreen ${ }^{\circledR}$ technology, is carried out on the exclusive basis of the binding properties of the selected candidates without the needs of performing a priori sequencing. It therefore enables the functional identification of high affinity aptamers. We validated the HAPIscreen (High throughput APtamer Identification screen) methodology using aptamers targeted to RNA hairpins, previously identified in our laboratory. We then screened pools of candidates issued from SELEX rounds in a 384 well microplate format and identify new RNA aptamers to pre-microRNAs.

Conclusions: HAPIscreen, an Alphascreen ${ }^{\circledR}$-based methodology for the identification of aptamers is faster and less biased than current procedures based on sequence comparison of selected oligonucleotides and sampling binding properties of few individuals. Moreover this methodology allows for screening larger number of candidates. Used here for selecting anti-premiR aptamers, HAPIscreen can be adapted to any type of tagged target and is fully amenable to automation.

\section{Background}

Aptamers are DNA, RNA or chemically-modified oligonucleotides selected from random pools of candidates containing up to $10^{15}$ different sequences on the basis of their ability to bind to other molecules [1-3] or to catalyze predetermined reactions $[4,5]$. Within these molecules, intra-molecular folding generates up to $10^{15}$ different structures that can be screened against a predetermined target for a chosen function, most often specific binding. Alternative steps of selection and amplification

\footnotetext{
* Correspondence: eric.chevet@u-bordeaux2.FR; jean-jacques.toulme@inserm. $\mathrm{fr}$

† Contributed equally

${ }^{1}$ Inserm U869, Institut Européen de Chimie et Biologie, 2 rue Robert Escarpit, 33706 Pessac, France

${ }^{2}$ Inserm U1053 Avenir, Bat 1A, 146 rue Léo Saignat, 33076 Bordeaux, France Full list of author information is available at the end of the article
}

of selected candidates progressively enrich the pool in sequences that are exquisitely adapted to the recognition of the molecule of interest. To date aptamers have been selected against many different types of targets: small organic compounds, proteins, nucleic acids and complex scaffolds such as intact viruses or live cells [6,7]. Aptamer molecules share essential properties with antibodies such as high affinity and specificity. In addition, aptamers offer an alternative for the recognition of molecules such as toxins against which no antibody can be easily raised or for use under conditions that lead to protein denaturation $[8,9]$. At last, aptamers that are chemically synthesized on solid supports can readily be conjugated to different pending groups making them versatile tools for the labelling or the detection of their cognate target. They are of high interest for analytical technology
C Biomed Central

C 2011 Dausse et al; licensee BioMed Central Ltd. This is an Open Access article distributed under the terms of the Creative Commons Attribution License (http://creativecommons.org/licenses/by/2.0), which permits unrestricted use, distribution, and reproduction in any medium, provided the original work is properly cited. 
[10-12] and numerous aptamer-based biosensors and probes have already been designed. These molecules are also of high potential value in medicine [13] as for instance an anti-VEGF aptamer has been recently approved by the Food and Drug Administration for the treatment of age-related macular degeneracy [14]. Several other molecules are currently being evaluated in clinical trials [15].

Aptamers are generally obtained by systematic evolution of ligands by exponential enrichment (SELEX) $[16,17]$ even though a selection process without any amplification step (non-SELEX) has been previously described $[18,19]$. The current approaches require sequencing of the selected clones at the end of the in vitro selection. This is followed by a limiting step relying on sequence comparison and individual evaluation of few candidates for the identification of aptamers (Figure 1). This methodology is slow, incompatible with automation and is strongly biased since efficiently amplified poor affinity binders may mask low copy/high affinity aptamer candidates.
Given the increasing demand for aptamers [13] it would therefore be more efficient to screen directly for the desired property i.e. affinity of the target for a candidate (Figure 1). To this end we developed a functional screen downstream of the SELEX pipeline that relies on the AlphaScreen ${ }^{\circledR}$ technology $[20,21]$. AlphaScreen ${ }^{\circledR}$ is based on the use of both Donor ( $D$, photosensitizer) and Acceptor (A, chemiluminescer) microbeads. Each bead is conjugated to one of the two potential interacting partners. A fluorescent signal is produced when both A and $\mathrm{D}$ beads are brought into proximity, thus reporting for the interaction between the biomolecular partners captured on the two beads [20]. This technology has been used for monitoring the interaction between various classes of molecules such as for instance protein/ protein [22-24] or protein/RNA interactions [25]. In the present report, we demonstrate the interest of HAPIscreen (High throughput APtamer Identification screen), an AlphaScreen ${ }^{\mathbb{B}}$-based method for the detection of aptamers targeted to different RNA hairpins of eukaryotic or viral origin.

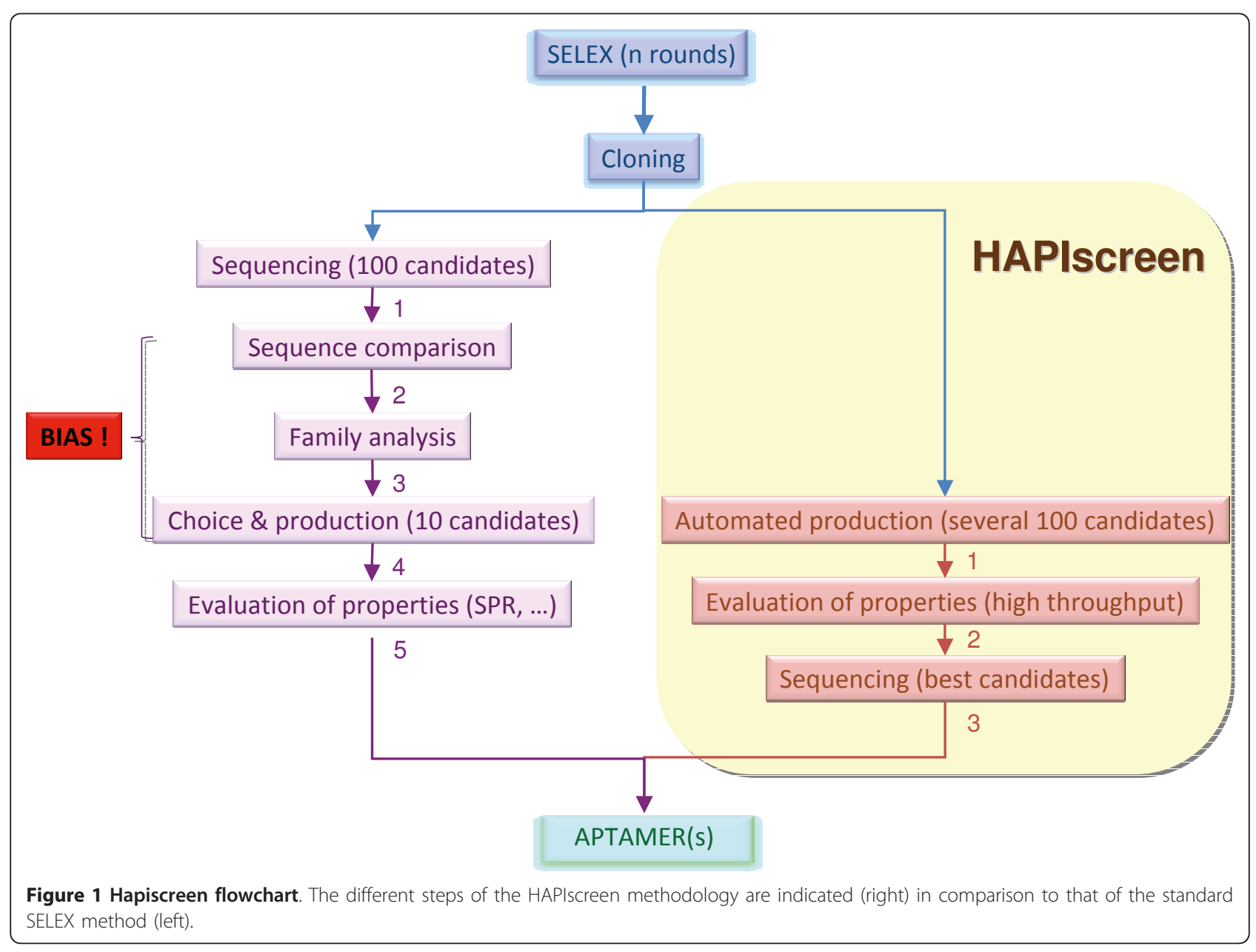




\section{Results and Discussion}

In the AlphaScreen ${ }^{\circledR}$ technology, Donor (D) and Acceptor (A) microbeads bear photosensitizing (phtalocyanin) and chemoluminescent molecules (rubrene), respectively. Laser excitation of the D beads at $680 \mathrm{~nm}$ causes ambient oxygen to be converted to the singlet state by phtalocyanin. Singlet oxygen species activate in turn a cascade of chemiluminescent reactions within the $\mathrm{A}$ beads leading to rubrene fluorescence detected between 520-620 nm using a photomultiplier tube-based microplate reader. A signal is produced when the $A$ and $D$ beads are brought into proximity $(<200 \mathrm{~nm})$ through the interaction between two molecules of interest respectively immobilized on the two beads [20] (Figure 2A). Therefore monitoring the emission signal allows for the detection of complex formation. We used this technology for monitoring the interactions between a candidate aptamer (RNA oligomer) and its target (RNA hairpin). In the assay described here, $\mathrm{D}$ beads are conjugated to the target and A beads to the candidate to be tested. This is repeated for a number of candidates issued from a given SELEX round and the emission specific to each pair is measured. Candidates generating a signal are picked and those corresponding to the strongest signal subsequently sequenced for further characterization (Figure 1).

To demonstrate the interest of HAPIscreen for the detection of aptamers targeted to different eukaryotic RNA hairpins, we undertook a proof of concept phase showing its applicability to monitor a previously characterized HIV-1 RNA-aptamer association and to evaluate pools of HCV RNA-aptamer complexes. This was followed by the integration of a selection/evaluation process into an automated-SELEX/AlphaScreen ${ }^{\circledR}$ pipeline allowing for identification of a large number of high affinity candidate aptamers to pre-microRNAs (premiRs).

\section{Monitoring RNA-RNA complexes with AlphaScreen ${ }^{\circledR}$}

In the proof of concept phase, we focused on a RNARNA complex that we previously characterized in great detail [26-28]. AlphaScreen ${ }^{\circledR}$ was used to quantify the interaction between the trans-activating responsive (TAR) RNA element of HIV-1 [29] and a RNA aptamer, R06 identified from a random library of oligonucleotides (Figure 2). These two oligoribonucleotides adopt a stemloop structure, display complementary sequences in the apical loop (Figure 2C) and were demonstrated to give rise to loop-loop (also called kissing) interactions. Increasing concentrations (0-40 $\mathrm{nM}$ ) of digoxigenincoupled R06 (dig-R06) were incubated in the presence of increasing concentrations of biotin-coupled TAR (biot-TAR), and constant amounts of streptavidin-D and anti-Dig-A beads. Such titration experiments were carried out at various biot-TAR concentrations ranging from 0 to $40 \mathrm{nM}$ (Figure 2B). This revealed typical bell- shaped AlphaScreen ${ }^{\circledR}$ signals showing a maximum for $40 \mathrm{nM}$ biot-TAR and $10 \mathrm{nM}$ dig-R06. Indeed as the anti-Dig-A-bead amount remains constant, addition of dig-R06 reaches a point at which it is no longer captured; consequently the excess of free dig-R06 competes with immobilized R06 for interacting with TAR captured on D-beads. This results in a decrease in fluorescence signal.

To demonstrate the specificity of the interaction, a competition assay was carried out in which increasing concentrations of unconjugated (free) R06 (fR06) were incubated in the presence of $10 \mathrm{nM}$ dig-R06 and $40 \mathrm{nM}$ biot-TAR (Figure 3A). As expected AlphaScreen ${ }^{\circledR}$ signal decrease correlated with increasing concentrations of fR06 added to the reaction, leading to an apparent IC50 of $16.7 \mathrm{nM} \pm 1.7$ (Figure 3B), a value reflecting an affinity in the same order of magnitude as that calculated using surface plasmon resonance (SPR) $[26,28]$.

Using thermal denaturation which was monitored by UV absorption spectroscopy, SPR and gel-shift assays, we previously showed that magnesium ions stabilized the TAR-R06 complex [26]. In addition, we also used the E. coli protein ROP, which is involved in the control of the ColE1 plasmid copy number and specifically recognizes loop-loop complexes. We previously demonstrated that ROP was able to bind to the TAR-R06 complex [30,31]. In the AlphaScreen ${ }^{\circledR}$ assay described above, increasing concentrations of ROP were added to biotTAR-dig-R06 complexes in the absence or in the presence of $3 \mathrm{mM} \mathrm{MgCl}$. The addition of $1 \mathrm{mM}$ ROP resulted in increased Alphascreen ${ }^{\mathbb{B}}$ signal indicating the formation of a highly stable ROP-R06-TAR kissing complex (Figure 3C). A $\sim 10$ fold higher AlphaScreen ${ }^{\circledR}$ signal was observed for this ternary complex in the presence of $3 \mathrm{mM} \mathrm{MgCl}{ }_{2}$ compared to no magnesium (not shown). These experiments demonstrate that this methodology provides signals correlated to the affinity of the complex. Indeed, no signal was detected for R06 variants that do not complex with TAR, whereas conditions known to increase the interaction (addition of magnesium or ROP protein) resulted in increased fluorescence signals. Collectively these results demonstrate that AlphaScreen ${ }^{\circledR}$ is suitable for monitoring the interaction between an aptamer and its target.

\section{Monitoring the evolution of SELEX RNA pools with HAPIscreen}

Our objective was then to adapt this approach to the screening of large pools of SELEX-derived sequences. To this end, it was necessary to capture every selected candidate on the A beads. The above methodology in which biotinylated candidates are used would no longer be easily adapted to such a goal. We rather considered the use of a biotinylated anchor complementary to a 


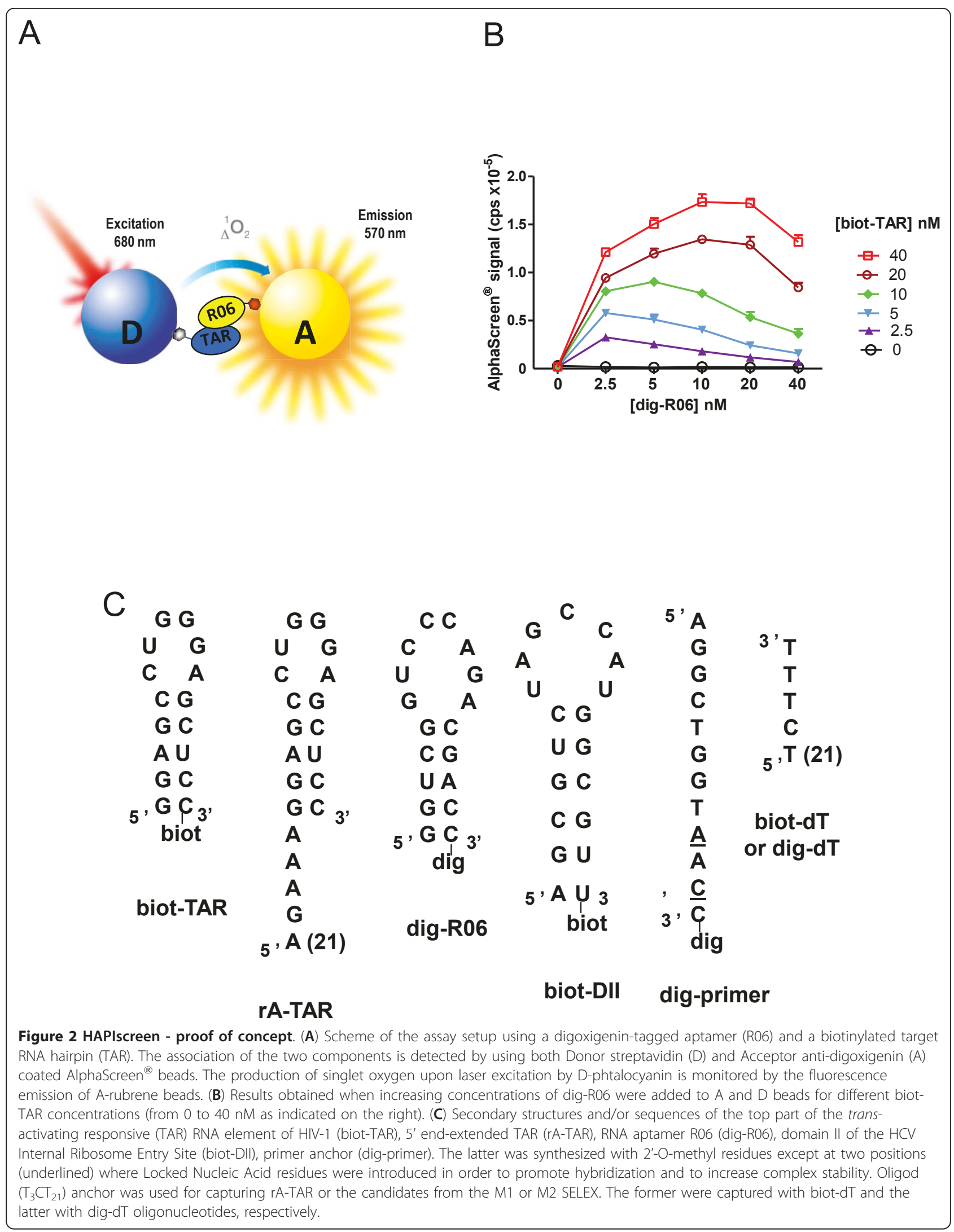




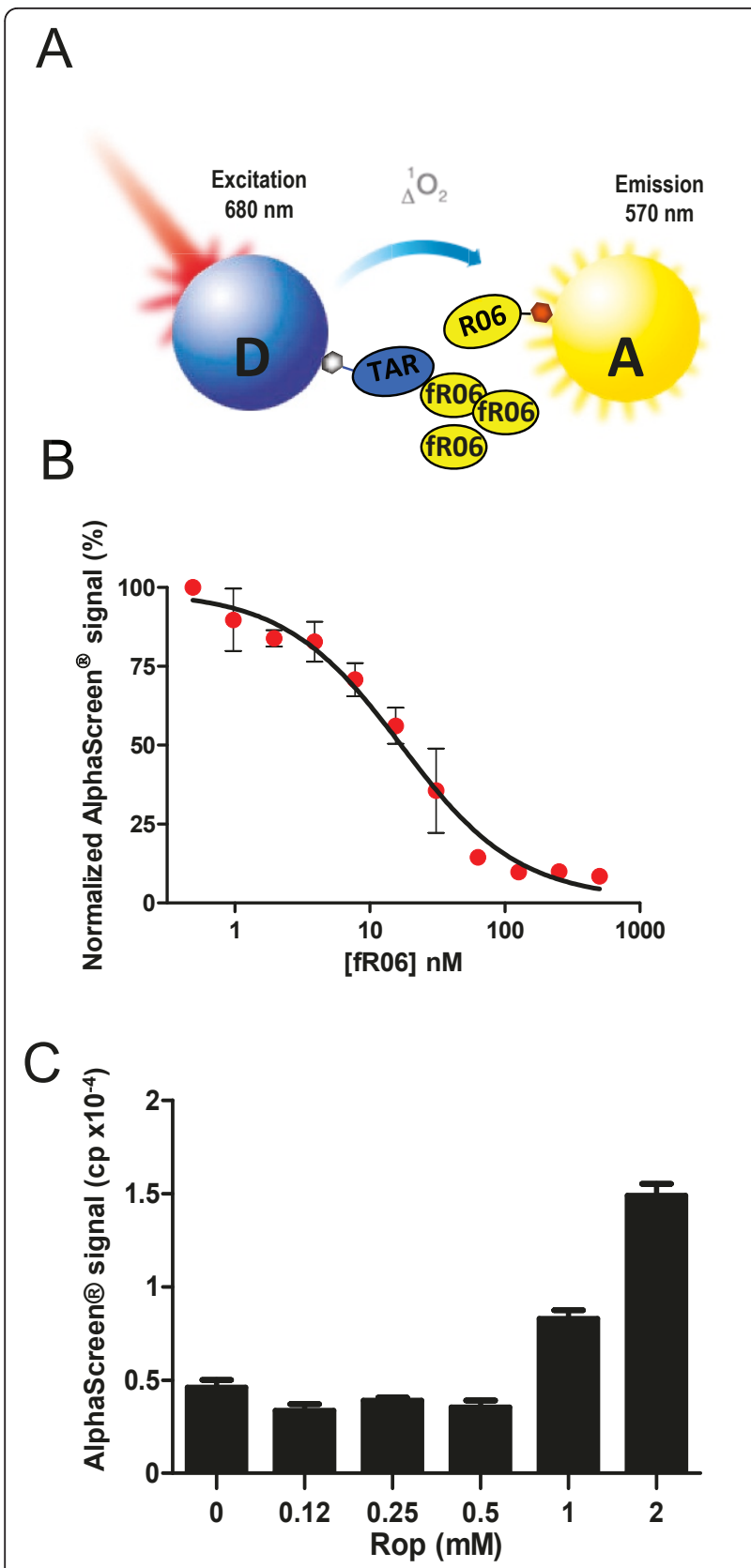

Figure 3 AlphaScreen ${ }^{\circledR}$-based characterization of TAR-R06 interaction. (A) Competition assay setup. The assay was carried out as described in Figure 2A in the presence of untagged R06 (fR06). (B) Competition assay performed as described in A. Increasing concentrations of fR06 were added to the reaction containing 10 nM of dig-R06 and 40 nM of biot-TAR and the AlphaScreen ${ }^{\circledR}$ signal was measured. Data are presented as percent of maximal signal (mean \pm SD) and are representative of at least 3 independent experiments carried out in triplicate. (C) Rop binding assay to the TAR-R06 kissing complex. Alphascreen ${ }^{\mathbb{B}}$ signal was obtained with a constant amount of biotinylated TAR and digoxiginated R06 (40 nM and $10 \mathrm{nM}$, respectively) as described in Materials and Methods, in the presence of increasing concentrations of the E. coli Rop protein. Data are representative of 3 independent experiments carried out in triplicate. pre-determined sequence on the candidates. Indeed every candidate contains fixed sequences flanking the random region, used in the SELEX process for the amplification of the selected candidates. An oligomer complementary to one of the flank efficiently allows for the capture of every candidate, at least those for which this region is not involved in a strong intra-molecular interaction. In order to validate this anchor-based approach a biotinylated oligod $\left(\mathrm{T}_{3} \mathrm{CT}_{21}\right)$ (biot-dT) (Figure $2 \mathrm{C}$ ) was assayed in the presence of $10 \mathrm{nM}$ dig-R06 and $40 \mathrm{nM}$ TAR bearing an oligor $\left(\mathrm{A}_{21} \mathrm{GA}_{3}\right)$ tail (rA-TAR) (Figure $2 \mathrm{C}$ ). Increasing concentrations of biot-dT (Figure 4A) led to a maximal AlphaScreen ${ }^{\circledR}$ signal for rA-TAR concentrations ranging from 40 to $80 \mathrm{nM}$, in accordance with a stoichiometric association between TAR and the biot-dT anchor and the respective Donor and Acceptor bead capture capacities under the current assay conditions. Therefore the anchor-based methodology allows for monitoring aptamer-target interactions.

We then used such a strategy for evaluating the evolution of populations derived from 7 rounds of RNA SELEX previously carried out in our laboratory against the domain II (DII) of the Hepatitis C Virus mRNA internal ribosome entry site. This domain that folds as a hairpin with a $7 \mathrm{nt}$ loop, was used as the target. In vitro selection against DII was previously demonstrated to produce high affinity aptamers [32]. To monitor the evolution of the RNA pool binding properties, candidates from the library (T0) and from rounds one to seven (T1-T7) were trapped on the acceptor beads using a digoxygenin-conjugated oligonucleotide, dig-primer (Figure 2C) complementary to their common 5 ' end. AlphaScreen ${ }^{\circledR}$ signals obtained with D-beads carrying a 19 nucleotide long hairpin corresponding to the top part of DII were detected from round 4 and further increased at subsequent rounds, thus indicating the progressive enrichment of the population in strong binders (Figure 4B) in agreement with band shift assays [32] and SPR experiments carried out with bimolecular complexes formed between the immobilized $\mathrm{HCV}$ target and the SELEX pools (Figure 4C). These results demonstrate that the AlphaScreen ${ }^{\circledR}$-based approach (HAPIscreen) could be undertaken for screening large collections of selected candidates using a unique anchor oligonucleotide.

\section{Identification of aptamers in RNA pools selected against premicroRNAs with HAPIscreen}

We then used HAPIscreen for evaluating the outcome of a SELEX experiment carried out using a RNA library against pre-microRNAs (premiRs). PremiRs display more or less perfect hairpin structures that are matured into functional miRs [33,34]. Aptamers raised against premiRs might consequently modulate miR interaction 


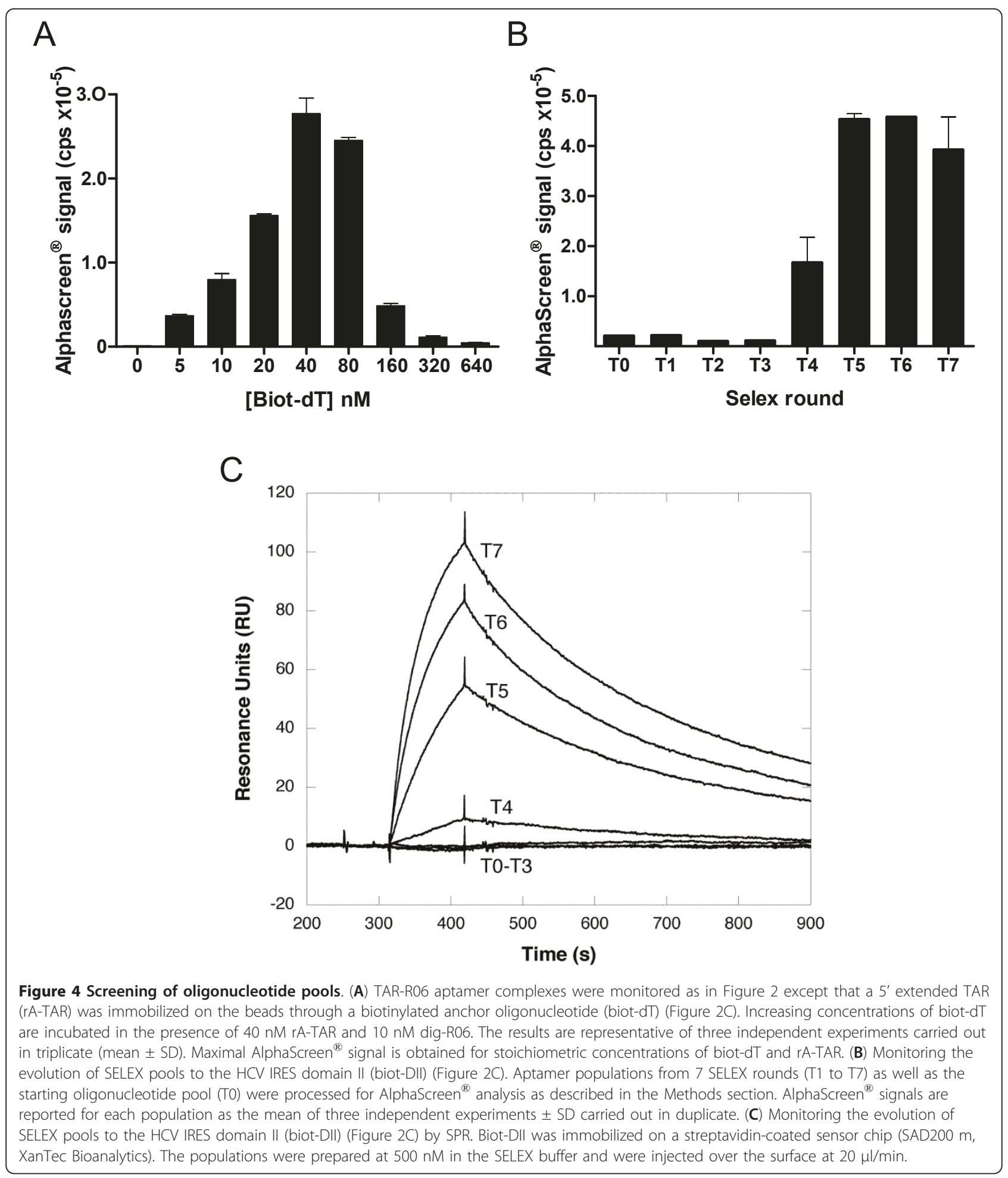

with proteins involved in the maturation process and impact on their regulatory function. Indeed oligonucleotides complementary to premiRs were shown to modulate the activity of miRs maturing enzymes [35,36]. SELEX was performed against two mixtures M1 and M2 of three human premiRs each (a, b, c and $x, y, z$, respectively) as described in Materials and Methods. At the end of seven SELEX rounds, the selected candidates were cloned and produced. Using HAPIscreen, candidates were trapped on the acceptor beads using digoxygenin-conjugated oligod 


\section{A}

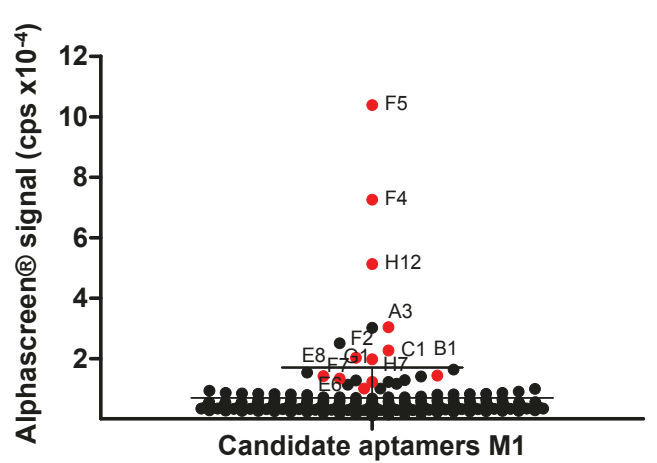

B

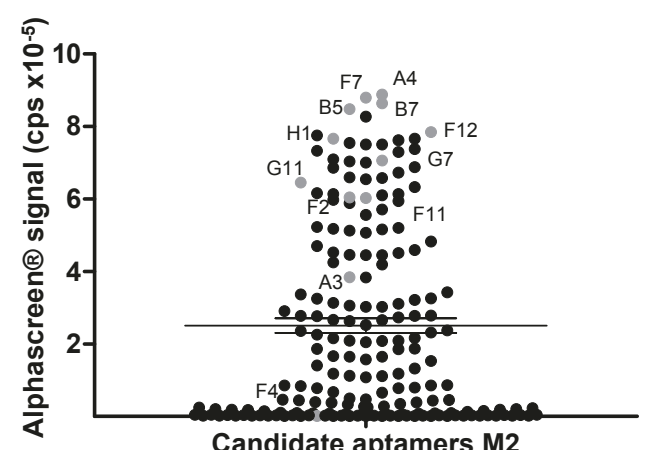

C

Candidate aptamers M2

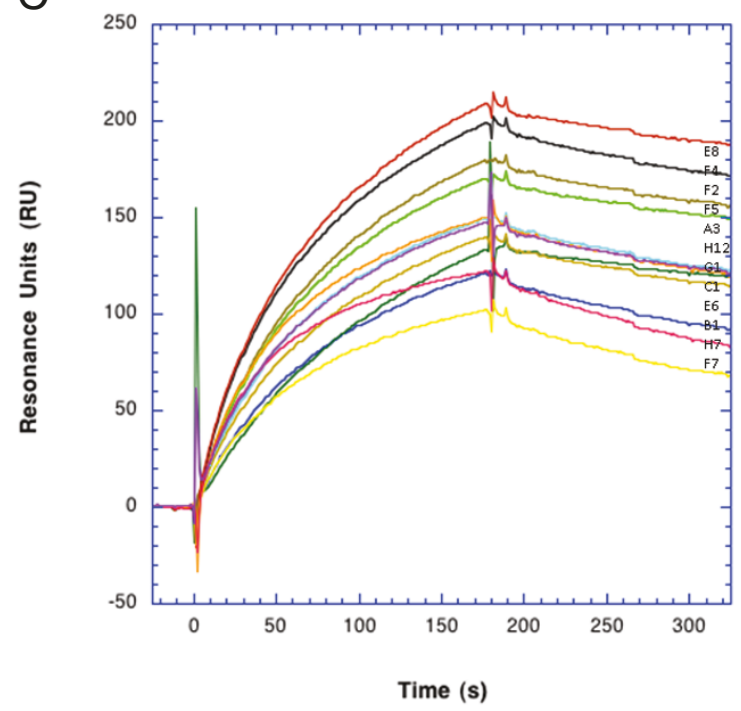

Figure 5 Screening of oligonucleotide pools. (A, B) AlphaScreen ${ }^{\circledR}$-based analysis of individual candidates issued from SELEX M1 against a mixed target population $\mathrm{a}, \mathrm{b}$ and $\mathrm{c}(\mathbf{A})$ or from SELEX M2 against the single target $\times(\mathbf{B})$. Error bars (horizontal bars in Figure $5 \mathrm{~A}, \mathrm{~B}$ ) represent the mean \pm SD values of three distinct AlphaScreen ${ }^{\mathbb{B}}$ signal measurement for each SELEX population. For M2 the 75 percentile above and below the average is shown. (C) SPR analysis of twelve RNA candidates (corresponding to the red dots in Figure 5A) binding to a premiR target from SELEX M1. 400 $\mathrm{RU}$ of the biotinylated target were immobilised on a streptavidincoated sensor chip (see Methods). The experiments were performed in the SELEX buffer at $23^{\circ} \mathrm{C}$ and the sensorgrams were collected at a flow rate of $20 \mu \mathrm{l} / \mathrm{min}$. Candidates were injected at a concentration of $500 \mathrm{nM}$.
$\left(\mathrm{T}_{3} \mathrm{CT}_{21}\right)$ (dig-dT) (Figure 2C) complementary to their identical 3' end and individually assayed against each individual biotin-tagged target. One hundred ninety-two clones derived from the 7th round-enriched populations against either M1 or M2 were screened in duplicate blindly against the mixture of the three baits $a, b$ and $c$ or against the premiR $\times$ alone, respectively. It should be pointed out that the second experiment aims at identifying the partner actually targeted by a given aptamer selected against the mixture; repeating this experiment with immobilized y or z would allow the complete assignment of aptamers and targets. These experiments were carried out in 384-well plates and led to the identification of hits within both RNA populations (Figures 5A, B). Candidates from each selection were picked according to their high AlphaScreen ${ }^{\circledR}$ signal (Figures 5A, B, red and grey dots, respectively) and tested individually by Surface Plasmon Resonance (Figure 5C). In the latter experiments, biotinylated pre-miRs a, b, c and $\mathrm{x}$ were individually immobilized on their respective sensor chip flow cells on which candidates were injected. Nine out of 12 (SELEX to $\times$ from M2) and 7 out of 12 (SELEX to a, b or c from M1) displayed evaluated $K_{D}$ values lower than or equal to $30 \mathrm{nM}$ for unique-based or mixture-based target assays, respectively. There was a fair agreement between both SPR and the Alphascreen ${ }^{\circledR}$ analyses even though the order of the signals was not rigorously the same with the two techniques (Figures 5A and 4C). We also tested the behaviour of 5 candidates that generated a low Alphascreen ${ }^{\circledR}$ signal using SPR. This revealed that 4 out of the 5 candidates gave a weak or no resonance signal (not shown).

High affinity aptamers identified by Alphascreen ${ }^{\circledR}$ were cloned and sequenced. As usual for in vitro selection we picked sequences displaying a high degree of similarity and identical motifs. Sequence differences likely account for the slight variation observed in Alphascreen ${ }^{\circledR}$ or SPR signals. Finally, using MFold [37], aptamers from SELEX against M2 were predicted to adopt a consensus hairpin structure with a loop containing several nucleotides complementary to pre-miR loops. This suggested the formation of loop-loop aptamer/premiR complexes as previously described for TAR aptamers [26]. These aptamers and the aptamer-premiRs complexes are presently being characterized and will be described in a forthcoming manuscript. Using a similar approach, aptamers derived from SELEX against M1 also showed sequences complementary to premiR target loop, allowing for the formation of 8 potential adjacent base pairs but were not predicted to fold as hairpins.

\section{Conclusion}

Herein, we have developed a method that overcomes the bias traditionally encountered in SELEX experiments. Usually candidates selected at the end of the process are 
cloned and sequenced. Sequences and/or predicted secondary structures are then compared in order to generate families and to choose few representatives that are then individually produced and characterized to identify aptamers (Figure 1). HAPIscreen bypasses the sequence comparison steps and directly allows for blind screening of aptamer candidates based on their exclusive binding properties rather than on sequence homologies. We demonstrate that HAPIscreen is a high throughput technology that can be used to analyze large collections of candidates. We used a 384-well plate format but HAPIscreen could as well be adapted to a 1536-well plate format. In addition, as the SELEX can be run simultaneously against a mixture of targets and the AlphaScreen $^{\circledR}$ analysis can be carried out against individual targets this predicts an increased discovery rate of aptamers. Moreover HAPIscreen is fully amenable to automation (currently the SELEX and the AlphaScreen ${ }^{(B)}$ steps are independently automated) and can be adapted for a wide range of targets due to the availability of different tags/beads. HAPIscreen also proved to be faster than traditional SELEX approaches as the process time was shortened by at least $50 \%$ from the isolated SELEX population to the identification of high affinity binders. The preparation of hundreds of candidates being achieved on an automated platform, this step is not time consuming. Finally, HAPIscreen potentially increases the chance of selecting orphan candidates (i.e. poorly amplified) by allowing the evaluation of larger aptamer collections. HAPIscreen therefore represents a major step forward in aptamer discovery and identification.

\section{Methods}

\section{Oligonucleotide synthesis and purification}

DNA primers and the biotinylated DNA anchor biot-dT, purchased either from Sigma or MWG Biotech, were purified by HPLC. All RNA targets and the digoxygenin 2'-O-methyl-LNA anchor (dig-primer) were chemically synthesized on an Expedite 8908 synthesizer (Applied Biosystems, USA) and purified by electrophoresis on denaturing $20 \%$ polyacrylamide, $7 \mathrm{M}$ urea gels. RNA candidates were synthesized by in vitro transcription using T7 RNA polymerase.

\section{AlphaScreen ${ }^{\circledR}$ assays}

The AlphaScreen ${ }^{\circledR}$ technology was used to assess the interaction between candidate oligonucleotides derived from SELEX experiments, and biotinylated target. Binding assays were performed using white 384-well Optiplates (Perkin Elmer) in a total volume of $25 \mu \mathrm{l}$. The AlphaScreen ${ }^{\circledR}$ reagents (anti-dig-coated Acceptor beads and streptavidin-coated Donor beads) were obtained from PerkinElmer. biot-TAR and dig-R06 (see figure 2C for oligonucleotide sequences) were prepared in a 10
$\mathrm{mM}$ sodium phosphate buffer, $\mathrm{pH} 7.2$ at $20^{\circ} \mathrm{C}$, containing $140 \mathrm{mM}$ potassium chloride, $20 \mathrm{mM}$ sodium chloride and $3 \mathrm{mM}$ magnesium chloride. Prior to the experiments the RNA samples were heated in this buffer at $95^{\circ} \mathrm{C}$ for $1 \mathrm{~min}$ and $30 \mathrm{~s}$ and cooled down on ice for 10 min. The protein ROP, purified as previously described [30], was prepared in this buffer with or without magnesium chloride. For the analysis of SELEX populations, denaturation and refolding of the candidate aptamers and targets prior to reaction with the anchor (biot-dT) was performed in water at $65^{\circ} \mathrm{C}$ for $3 \mathrm{~min}$ or $80^{\circ} \mathrm{C}$ for $1 \mathrm{~min}$, respectively. After denaturation, candidate aptamer and target were quickly cooled down to $4^{\circ}$ $\mathrm{C}$ for $3 \mathrm{~min}$ and then equilibrated at room temperature (RT) for $5 \mathrm{~min}$ before adding the selection buffer (20 $\mathrm{mM}$ sodium acetate, $140 \mathrm{mM}$ potassium acetate, $3 \mathrm{mM}$ magnesium acetate, $20 \mathrm{mM}$ HEPES; $\mathrm{pH}$ 7.4). Equal volumes $(5 \mu \mathrm{l})$ of each partner, candidate and target, were incubated for $45 \mathrm{~min}$ at room temperature (RT), at final concentrations of $0.2 \mu \mathrm{M}$ and $0.625 \mu \mathrm{M}$, respectively. In parallel Acceptor beads $(20 \mu \mathrm{g} / \mathrm{ml})$ were incubated with dig-primer $(0.625 \mu \mathrm{M})$ for $1 \mathrm{~h}$ at room temperature in the selection buffer. Then, $10 \mu \mathrm{l}$ of each of the interacting partners were added to the plate, after 45 -min incubation at RT, $5 \mu$ of Donor beads at a 20 $\mu \mathrm{g} / \mathrm{ml}$ concentration were added to the mixture. All manipulations involving AlphaScreen ${ }^{\circledR}$ beads were performed under subdued lighting. The plates were allowed to incubate either $1 \mathrm{~h}$ or overnight in the dark at room temperature. Light signal was detected by using an EnVision ${ }^{\circledR}$ multilabel plate reader from PerkinElmer.

\section{In vitro selection}

The RNA library used for the selections was obtained by transcription of the DNA library (5'-GTGTGACCG ACCGTGGTGC-N30-GCAGTGAAGGCTGGTAACC3') as previously described [38]. Two different primers P20 5'-GTGTGACCGACCGTGGTGC-3' and 3'SL containing the $\mathrm{T} 7$ transcription promoter (underlined) 5'TAATACGACTCACTATAGGTTACCAGCCTTCA CTGC-3' were used for PCR amplification. Oligonucleotide P20 was also used to prime reverse transcription. Selection steps were performed in the SELEX buffer $(20$ $\mathrm{mM}$ HEPES, $\mathrm{pH} 7.4$ at $23^{\circ} \mathrm{C}, 20 \mathrm{mM}$ sodium acetate, $140 \mathrm{mM}$ potassium acetate and $3 \mathrm{mM}$ magnesium acetate) at $23^{\circ} \mathrm{C}$ on an in-house assembled automated workstation (Tecan Freedom EVO 150). All steps (magnetic bead separation, vacuum purification, PCR amplification and transcription) were carried out in microplates. Two parallel SELEX, each against 3 target premiRs, constituting mixtures M1 and M2, were performed on the automated workstation. For each SELEX, $3 \mu \mathrm{M}$ of the RNA library was heated at $80^{\circ} \mathrm{C}$ for $1 \mathrm{~min}$, cooled at $4^{\circ} \mathrm{C}$ for $3 \mathrm{~min}$, placed at room temperature for $5 \mathrm{~min}$ and mixed 
for the counter-selection with streptavidin-coated beads (50 $\mu \mathrm{g}$ of Streptavidin MagneSphere ${ }^{\circledR}$ Paramagnetic Particles from Promega or $500 \mu \mathrm{g}$ of Dynabeads M-280). RNA candidates not retained by the beads were then mixed and incubated for 10 min with 10 pmol of 3 different 3' end biotinylated premiRs that were previously immobilized on streptavidin beads. Unbound RNA was removed and the beads were washed twice with $100 \mu \mathrm{l}$ of the SELEX buffer. The bound RNA candidates were eluted from the premiRs by heating for $1 \mathrm{~min}$ at $75^{\circ} \mathrm{C}$ in $50 \mu \mathrm{l}$ of water. RNA candidates were reverse-transcribed with 200 units of M-MLV reverse transcriptase RNase $\mathrm{H}^{-}$Point mutant (Promega) for $50 \mathrm{~min}$ at $50^{\circ} \mathrm{C}$. The cDNA was amplified by PCR at $63^{\circ} \mathrm{C}$ with 20 units of the DNA polymerase AmpliTaq Gold ${ }^{\mathrm{TM}}$ (PerkinElmer) and the two primers P20 and 3'SL at $2 \mu \mathrm{M}$, during 25 cycles. RNA candidates were obtained by in vitro transcription of the PCR products with the Ampliscribe T7 transcription kit from Epicentre Biotechnologies. After 2 first manual and 5 automated rounds of selection against pre-miRs, carried out on an EVO150 (Tecan) in house-assembled robot, selected candidates were cloned using the TOPO TA cloning kit (Invitrogen).

\section{Synthesis, capture and sequencing of the candidates}

In order to generate candidates for high throughput screening, we set up a second automated workstation (Tecan Freedom EVO 200) equipped with a thermal cycler, an orbital shaker, a magnetic particle separation module and a vacuum separation module. Three hundred and eighty four clones (192 clones from either M1 or M2 populations) from round 7 were produced blindly on this second workstation. Candidates were directly amplified from colonies with a $5^{\prime}$ end $\operatorname{oligod}\left(\mathrm{T}_{21} \mathrm{CT}_{3}\right)$ (underlined) lengthened P20 5'-TTTTTTTTTTTTTTT TTTTTTCTTTGTGTGACCGACCGTGGTGC-3' and the 3'SL 5'-TAATACGACTCACTATAGGTTACCAGCCTTCACTGC-3' primers allowing the addition of an oligodA/T extension to the PCR products. RNAs produced by transcription of these PCR amplifications contained a 3' end oligorA tail that was used to capture them for the AlphaScreen ${ }^{\circledR}$ tests with a digoxygeninconjugated oligonucleotide (dig-dT) (Figure 2C). Candidates were sequenced using the BigDye Terminator v1.1 cycle sequencing kit (Applied Biosystems) according to the manufacturer's instructions.

\section{Surface Plasmon Resonance analyses}

SPR experiments were performed at $23^{\circ} \mathrm{C}$ with a BIAcore $^{\mathrm{TM}} 3000$ apparatus. The biotinylated premiRs were immobilised at $50 \mathrm{nM}$ (300 to $400 \mathrm{RU}$ ) on SA (BIAcore, GE Heathcare Life Sciences; Sweden) or SAD200 m sensor chips (XanTech Bioanalytics; Germany) coated with streptavidin. Aptamers were injected at $500 \mathrm{nM}$ in the
SELEX buffer at a $20 \mu \mathrm{l} / \mathrm{min}$ flow rate. After each injection of the candidates, the target-surface was regenerated with a 1 min pulse of a mixture containing $40 \%$ formamide, $30 \mathrm{mM}$ EDTA and 3.6 $\mathrm{M}$ urea prepared in milli-Q water. The sensorgrams were analysed with the BIAeval software 4.1 as previously described [30]. Sensorgrams were double-referenced [39].

\section{Acknowledgements}

We are grateful to Ms E. Daguerre (Inserm U869) for skilled technical assistance and N. Pierre (Inserm U869) for oligonucleotides synthesis. We are indebted to Dr S. Da Rocha Gomes for RNA SELEX against the domain II of the HCV mRNA internal ribosome entry site. We thank Drs J. Rosenbaum and F. Darfeuille (Bordeaux), M. Gait and M. Fabani (Cambridge) for critically reading the manuscript. This work was supported by the "Conseil Régional d'Aquitaine" to JJT; and by Inserm (Avenir); Institut National du Cancer; "Conseil Régional d'Aquitaine"; and Fondation pour la Recherche Médicale to EC.

\section{Author details}

'Inserm U869, Institut Européen de Chimie et Biologie, 2 rue Robert Escarpit, 33706 Pessac, France. ${ }^{2}$ Inserm U1053 Avenir, Bat 1A, 146 rue Léo Saignat, 33076 Bordeaux, France. ${ }^{3}$ Université de Bordeaux, 146 rue Léo Saignat, 33076 Bordeaux, France.

\section{Authors' contributions}

ED carried out, designed and analyzed the SELEX experiments. ST designed and performed the Alphascreen ${ }^{\circledR \oplus}$ experiments and analyzed the data. LE performed the manual and automated selection. CDP designed the interaction models and participated in the SPR measurements. EC supervised the Alphascreen ${ }^{{ }^{\circledR}}$ measurements. JJT conceived the study and participated in its coordination. EC and JJT drafted the manuscript. All authors read and approved the final manuscript.

\section{Competing interests}

The authors declare that they have no competing interests.

Received: 5 January 2011 Accepted: 3 June 2011 Published: 3 June 2011

ReferencesIn vitro selection of RNA molecules that bind specific ligandsEllington AD, Szostak JW: In vitro selection of RNA molecules that bind specific ligands. Nature 1990, 346:818-822.

2. Robertson DL, Joyce GF: Selection in vitro of an RNA enzyme that specifically cleaves single-stranded DNA. Nature 1990, 344:467-468

3. Tuerk C, Gold L: Systematic evolution of ligands by exponential enrichment: RNA ligands to bacteriophage T4 DNA polymerase. Science 1990, 249:505-510

4. Bartel DP, Szostak JW: Isolation of new ribozymes from a large pool of random sequences [see comment]. Science 1993, 261:1411-1418.

5. Lehman N, Joyce GF: Evolution in vitro of an RNA enzyme with altered metal dependence. Nature 1993, 361:182-185.

6. Dausse E, Da Rocha Gomes S, Toulmé JJ: Aptamers: a new class of oligonucleotides in the drug discovery pipeline? Curr Opin Pharmacol 2009, 9:602-607.

7. Hall B, Arshad S, Seo K, Bowman C, Corley M, Jhaveri SD, Ellington AD: In vitro selection of RNA aptamers to a protein target by filter immobilization. Curr Protoc Mol Biol 2009, 24:23, Unit 24.

8. Tok JB, Fischer NO: Single microbead SELEX for efficient ssDNA aptamer generation against botulinum neurotoxin. Chem Commun (Camb) 2008, 1883-1885.

9. Cruz-Aguado JA, Penner G: Determination of ochratoxin a with a DNA aptamer. J Agric Food Chem 2008, 56:10456-10461.

10. Yan AC, Levy M: Aptamers and aptamer targeted delivery. RNA Biol 2009, 6.

11. Tombelli S, Mascini M: Aptamers as molecular tools for bioanalytical methods. Curr Opin Mol Ther 2009, 11:179-188.

12. Mairal T, Cengiz Ozalp V, Lozano Sanchez P, Mir M, Katakis I, O'Sullivan CK: Aptamers: molecular tools for analytical applications. Anal Bioanal Chem 2007, 390:989-1007. 
13. Keefe $A D$, Pai $S$, Ellington $A$ : Aptamers as therapeutics. Nat Rev Drug Discov 2010, 9:537-550.

14. Ng EW, Shima DT, Calias P, Cunningham ET Jr, Guyer DR, Adamis AP: Pegaptanib, a targeted anti-VEGF aptamer for ocular vascular disease. Nat Rev Drug Discov 2006, 5:123-132.

15. Bunka DH, Platonova O, Stockley PG: Development of aptamer therapeutics. Curr Opin Pharmacol 2010, 10:557-562.

16. Gold L, Brown D, He Y, Shtatland T, Singer BS, Wu Y: From oligonucleotide shapes to genomic SELEX: novel biological regulatory loops. Proc Natl Acad Sci USA 1997, 94:59-64.

17. Gopinath SC: Methods developed for SELEX. Anal Bioanal Chem 2007, 387:171-182.

18. Berezovski MV, Musheev MU, Drabovich AP, Jitkova JV, Krylov SN: NonSELEX: selection of aptamers without intermediate amplification of candidate oligonucleotides. Nat Protoc 2006, 1:1359-1369.

19. Javaherian S, Musheev MU, Kanoatov M, Berezovski MV, Krylov SN: Selection of aptamers for a protein target in cell lysate and their application to protein purification. Nucleic Acids Res 2009, 37:e62

20. Taouji S, Dahan S, Bosse R, Chevet E: Current Screens Based on the AlphaScreen Technology for Deciphering Cell Signalling Pathways. Curr Genomics 2009, 10:93-101.

21. Eglen RM, Reisine T, Roby P, Rouleau N, Illy C, Bosse R, Bielefeld M: The use of AlphaScreen technology in HTS: current status. Curr Chem Genomics 2008, 1:2-10.

22. Bouchecareilh M, Caruso ME, Roby P, Parent S, Rouleau N, Taouji S, Pluquet O, Bosse R, Moenner M, Chevet E: AlphaScreen-based characterization of the bifunctional kinase/RNase IRE1alpha: a novel and atypical drug target. J Biomol Screen 2010, 15:406-417.

23. Arcand M, Roby P, Bosse R, Lipari F, Padros J, Beaudet L, Marcil A, Dahan S: Single-well monitoring of protein-protein interaction and phosphorylation-dephosphorylation events. Biochemistry 2010, 49:3213-3215

24. Caruso ME, Jenna S, Beaulne $S$, Lee EH, Bergeron A, Chauve C, Roby P Rual JF, Hill DE, Vidal M, et al: Biochemical clustering of monomeric GTPases of the Ras superfamily. Mol Cell Proteomics 2005, 4:936-944.

25. Mills NL, Shelat AA, Guy RK: Assay Optimization and Screening of RNAProtein Interactions by AlphaScreen. J Biomol Screen 2007, 12:946-955.

26. Ducongé F, Di Primo C, Toulmé JJ: Is a closing "GA pair" a rule for stable loop-loop RNA complexes? J Biol Chem 2000, 275:21287-21294

27. Van Melckebeke H, Devany M, Di Primo C, Beaurain F, Toulmé JJ, Bryce DL, Boisbouvier J: Liquid-crystal NMR structure of HIV TAR RNA bound to its SELEX RNA aptamer reveals the origins of the high stability of the complex. Proc Natl Acad Sci USA 2008, 105:9210-9215.

28. Lebars I, Legrand P, Aimé A, Pinaud N, Fribourg S, Di Primo C: Exploring TAR-RNA aptamer loop-loop interaction by X-ray crystallography, UV spectroscopy and surface plasmon resonance. Nucleic Acids Res 2008, 36:7146-7156.

29. Bannwarth S, Gatignol A: HIV-1 TAR RNA: the target of molecular interactions between the virus and its host. Curr HIV Res 2005, 3:61-71.

30. Di Primo C, Lebars I: Determination of refractive index increment ratios for protein-nucleic acid complexes by surface plasmon resonance. Anal Biochem 2007, 368:148-155.

31. Watrin M, Von Pelchrzim F, Dausse E, Schroeder R, Toulmé JJ: In vitro selection of RNA aptamers derived from a genomic human library against the TAR RNA element of HIV-1. Biochemistry 2009, 48:6278-6284.

32. Da Rocha Gomes S, Dausse E, Toulmé JJ: Determinants of apical loopinternal loop RNA-RNA interactions involving the HCV IRES. Biochem Biophys Res Commun 2004, 322:820-826.

33. Zeng Y: Principles of micro-RNA production and maturation. Oncogene 2006, 25:6156-6162.

34. Chekulaeva M, Filipowicz W: Mechanisms of miRNA-mediated posttranscriptional regulation in animal cells. Curr Opin Cell Biol 2009, 21:452-460.

35. Michlewski G, Guil S, Semple CA, Caceres JF: Posttranscriptional regulation of miRNAs harboring conserved terminal loops. Mol Cell 2008, 32:383-393.

36. Lunse CE, Michlewski G, Hopp CS, Rentmeister A, Caceres JF, Famulok M, Mayer G: An aptamer targeting the apical-loop domain modulates primiRNA processing. Angew Chem Int Ed Engl 2010, 49:4674-4677.

37. Zuker M: Mfold web server for nucleic acid folding and hybridization prediction. Nucleic Acids Res 2003, 31:3406-3415.
38. Dausse E, Cazenave C, Rayner B, Toulmé JJ: In vitro selection procedures for identifying DNA and RNA aptamers targeted to nucleic acids and proteins. Methods Mol Biol 2005, 288:391-410.

39. Myszka DG: Improving biosensor analysis. J Mol Recognit 1999, 12:279-284.

doi:10.1186/1477-3155-9-25

Cite this article as: Dausse et al.: HAPIscreen, a method for highthroughput aptamer identification. Journal of Nanobiotechnology 2011 9:25.

\section{Submit your next manuscript to BioMed Central and take full advantage of:}

- Convenient online submission

- Thorough peer review

- No space constraints or color figure charges

- Immediate publication on acceptance

- Inclusion in PubMed, CAS, Scopus and Google Scholar

- Research which is freely available for redistribution

Submit your manuscript at www.biomedcentral.com/submit
Biomed Central 